\title{
In vitro susceptibility of Staphylococcus aureus and Staphylococcus epidermidis isolated from prosthetic joint infections
}

\author{
Diana Molina-Manso ${ }^{1}$, Gema del Prado ${ }^{1}$, Alberto Ortiz-Pérez ${ }^{1}$, Miguel Manrubia-Cobo ${ }^{1}$, \\ Enrique Gómez-Barrena ${ }^{2}$, José Cordero-Ampuero ${ }^{3}$ and Jaime Esteban ${ }^{1}$
}

Prosthetic joint infections (PJI) are severe complications in Orthopedics, with Staphylococcus aureus and Staphylococcus epidermidis being the most commonly isolated pathogens. The variable antimicrobial susceptibility found in these microorganisms, along with the increasing number of methicillin-resistant strains, increases the difficulty of antibiotic selection and makes it necessary to perform individual susceptibility studies to select the optimal antibiotic treatment. The aim of this study was to evaluate the in vitro susceptibility pattern of 35 clinical strains isolated from PJI (17 S. aureus and 18 S. epidermidis) against rifampin, vancomycin, tygecicline, clindamycin, cotrimoxazole, cloxacillin, ciprofloxacin, daptomycin and fosfomycin. In vitro susceptibility assays were performed using the broth microdilution method and agar dilution for fosfomycin. MBC was also determined. Tygecicline and daptomycin showed the highest antimicrobial activity with low MIC 90 values, and no resistant strains were detected. On the other hand, ciprofloxacin and cloxacillin exhibited a poor antimicrobial effect with a high percentage of nonsusceptible strains in both species. Bactericidal activity rates revealed the bacteriostatic behavior of rifampin, tygecicline, cotrimoxazole, fosfomycin and clindamycin, whereas vancomycin and cloxacillin showed species- and strain-dependent behavior. Daptomycin and ciprofloxacin were observed to be efficient bactericidal agents against the tested strains. According to our data, rifampin, tigecycline, daptomycin and fosfomycin showed high in vitro activity against most staphylococcal strains isolated from the PJIs tested, although daptomycin seems to be the best alternative to vancomycin therapy.

The Journal of Antibiotics (2012) 65, 505-508; doi:10.1038/ja.2012.62; published online 1 August 2012

Keywords: infection; joint; prostheses; Staphylococcus; susceptibility

\section{INTRODUCTION}

The use of joint prostheses has become an extremely important advance in modern medicine because it has helped many patients to improve their quality of life. Nevertheless, prosthetic joint infection (PJI) is a rare but severe complication related to these procedures. ${ }^{1,2}$ It is well known that bacterial infection after prosthesis implantation causes high morbidity and even mortality among the affected patients. Moreover, these infections frequently require prosthesis removal and a prolonged antibiotic treatment to cure the patients and ensure complete recovery. 3,4

Although a wide range of bacterial species can cause PJI, Staphylococcus aureus and Staphylococcus epidermidis are the most commonly isolated pathogens from implants, representing about $2 / 3$ of the cases. ${ }^{6}$ The increasing number of methicillin-resistant $S$. aureus (MRSA) and methicillin-resistant S. epidemidis (MRSE) pathogens is a matter of special concern, as this greater prevalence increases the difficulty of antibiotic selection. ${ }^{7}$ Both MRSA and MRSE strains are also usually resistant to other antibiotics, and although vancomycin remains the elective therapy for these cases, its efficacy has been declining over the last few years. ${ }^{8,9}$ For this reason, other antibiotics have recently been considered as alternatives to vancomycin. ${ }^{10-15}$ Despite these facts, antimicrobial susceptibility remains extremely variable among these microorganisms, making it necessary to perform individual susceptibility studies for each strain to select the best antibiotic combination for treatment. ${ }^{16}$

The aim of this study was to evaluate the in vitro susceptibility pattern of $S$. aureus and S. epidermidis strains isolated from retrieved prosthetic joint implants against antibiotics commonly used in the treatment of PJI.

\section{MATERIALS AND METHODS}

Bacterial strains

Thirty-five clinical Staphylococcus isolates (17 S. aureus and 18 S. epidermidis) as well as two biofilm-producing collection strains, S. aureus 15981

${ }^{1}$ Department of Clinical Microbiology, IIS-Fundación Jiménez Díaz, Madrid, Spain; ${ }^{2}$ diPaz-Hospital de La Paz, Institute for Health Research, Madrid, Spain and ${ }^{3}$ Department of Orthopaedics, Hospital Universitario de La Princesa, Madrid, Spain

Correspondence: Dr J Esteban, Department of Clinical Microbiology, IIS- Fundación Jiménez Díaz, Av. Reyes Católicos 2, Madrid 28040, Spain.

E-mail: jestebanmoreno@gmail.com

Received 1 March 2012; revised 19 April 2012; accepted 28 June 2012; published online 1 August 2012 
(Valle et al. ${ }^{17}$ ) and S. epidermidis ATCC 35984, were included. Clinical strains were isolated from orthopedic devices retrieved from patients with diagnosed PJI (one strain per patient) using a previously described protocol ${ }^{18}$ between January 2007 and December 2010. Species identification was performed by using the API Staph System (bioMérieux, France). Three S. epidermidis clinical strains were identified as small colony variant strains (SCV). All the strains tested were cultured and kept frozen in skim milk at $-80^{\circ} \mathrm{C}$ until the experiments were performed.

More details about the tested strains are shown in Table 1.

\section{Antibiotics}

Nine antimicrobial agents were selected for susceptibility testing: rifampin, vancomycin, ciprofloxacin, cotrimoxazole, cloxacillin, clindamycin, (Sigma, Munich, Germany), tygecicline (Pfizer, New York, NY, USA), daptomycin (Novartis, Basel, Switzerland) and fosfomycin (ERN, Barcelona, Spain). The antibiotics were prepared according to the instructions published by the Clinical and Laboratory Standards Institute $(\mathrm{CLSI})^{19}$ and kept frozen at $-20{ }^{\circ} \mathrm{C}$ until the experiments were performed.

\section{Susceptibility test}

In vitro susceptibility assays were performed using the broth microdilution method as described by the CLSI. ${ }^{19}$ In the case of fosfomycin, agar dilution was used together with broth microdilution. A calcium supplement $\left(\mathrm{CaCl}_{2}\right.$ $50 \mathrm{mgl}^{-1}$ of final concentration) was added for daptomycin in vitro susceptibility testing. Fosfomycin was tested at the dilution range of 0.06-128 $\mathrm{mgl}^{-1}$; vancomycin, cotrimoxazole, cloxacillin and daptomycin from 0.015 to $32 \mathrm{mgl}^{-1}$; tygecicline, clindamycin and ciprofloxacin from 0.004 to $8 \mathrm{mgl}^{-1}$; and rifampin from 0.001 to $4 \mathrm{mgl}^{-1}$. For MIC determinations, plates were incubated for $24 \mathrm{~h}$, and for the three S. epidermidis SCV strains, the incubation time was extended to $48 \mathrm{~h}$. $\mathrm{MIC}_{50}, \mathrm{MIC}_{90}$ and antibiotic non-susceptibility rates were calculated attending to the susceptibility breakpoints published by the European Committee on Antimicrobial Susceptibility Testing (EUCAST) and CLSI ${ }^{19,20}$

Table 1 Bacterial strains

\begin{tabular}{|c|c|c|c|c|c|}
\hline \multicolumn{3}{|c|}{ S. aureus } & \multicolumn{3}{|c|}{ S. epidermidis } \\
\hline Strain & Type & $\begin{array}{c}\text { Source of } \\
\text { isolation }\end{array}$ & Strain & Type & $\begin{array}{c}\text { Source of } \\
\text { isolation }\end{array}$ \\
\hline 15981 & Collection & - & $\begin{array}{c}\text { ATCC } \\
35984\end{array}$ & Collection & - \\
\hline P1 & Clinical & OS & P6. 5 & Clinical & NS \\
\hline P2 & Clinical & HPC & P23.2 & Clinical & HPC \\
\hline P4 & Clinical & HPC & P33.1 & Clinical & HPC \\
\hline P18 & Clinical & HPC & P53B & Clinical & HPC \\
\hline P19 & Clinical & HPC & P55 & Clinical & HPC \\
\hline P41 & Clinical & OS & P61.T1 & Clinical & NS \\
\hline P61.T3 & Clinical & NS & P61.T2 & Clinical & NS \\
\hline P61.T4 & Clinical & NS & P74 & Clinical & HPC \\
\hline P62.A & Clinical & OS & P101 & Clinical & HPC \\
\hline P68 & Clinical & OS & P146.G & Clinical & NS \\
\hline P82.1 & Clinical & OS & P146.B & Clinical & NS \\
\hline P95 & Clinical & NS & P194 & Clinical & NS \\
\hline P104.1 & Clinical & OS & P289.1 & Clinical & HPC \\
\hline P112 & Clinical & os & P289.2 & Clinical & HPC \\
\hline P138 & Clinical & HPC & P289.3 & Clinical & HPC \\
\hline P251 & Clinical & NS & P223 & Clinical & HPC \\
\hline P272.1 & Clinical & KPC & P236 & Clinical & KPC \\
\hline & & & P281 & Clinical & KPC \\
\hline
\end{tabular}

Abbreviations: HPC, hip prostheses components; KPC, knee prostheses components; NS, nails and screws; OS, osteosynthesis materials.
MBC was calculated by colony counting in trypcase soy agar $+5 \%$ sheep blood plates after direct plating of the wells-content without visible microbial growth and interpreted according to an internationally accepted definition. ${ }^{21}$

\section{RESULTS}

Table 2 shows the antibiotic susceptibility assay results: MIC $_{50}$ and $\operatorname{MIC}_{90}\left(\mathrm{mgl}^{-1}\right)$ as well as the percentage of nonsusceptible bacterial strains.

Tygecicline and daptomycin showed the highest levels of antimicrobial activity with low $\mathrm{MIC}_{90}$ values $\left(0.5\right.$ and $1 \mathrm{mgl}^{-1}$ for $S$. aureus and 0.25 and $1 \mathrm{mgl}^{-1}$ for $S$. epidermidis, respectively) and no resistant strains were detected.

Fosfomycin also exhibited substantial activity against both bacterial species, with only one $S$. aureus nonsusceptible strain $(5.56 \%$ of the total of $S$. aureus). Rifampin and vancomycin showed high activity, with low rates of nonsusceptibility. Cotrimoxazole and clindamycin showed higher activity for $S$. aureus than S. epidermidis (5.56 and $11.11 \%$, respectively).

Ciprofloxacin and cloxacillin exhibited a poor antimicrobial effect with a high percentage of nonsusceptible strains in both bacterial species. The high number of MRSA strains in this series is especially remarkable, and was also correlated with $S$. aureus ciprofloxacin nonsusceptibility.

On the other hand, bactericidal activity rates (also shown in Table 2) revealed the bacteriostatic behavior of rifampin, tygecicline, cotrimoxazole, fosfomycin and clindamycin for most strains.

Vancomycin showed a higher bactericidal effect against $S$. epidermidis than against $S$. aureus $(94.74 \%$ vs $55.56 \%$, respectively), showing strain-dependent behavior. This strain-dependent effect appears also for cloxacillin (50\% in both species).

The only two antibiotics that showed bactericidal activity for most strains of both species were ciprofloxacin and daptomycin, both with similar bactericidal activity rates.

\section{DISCUSSION}

Treatment of PJI caused by $S$. aureus and $S$. epidermidis is still a challenge because of the variable behavior that these microorganisms exhibit against the different antibiotics available and the increasing number of multidrug-resistant strains seen over recent years. ${ }^{22}$ Efficacy in both diagnosis and treatment of such staphylococcal PJIs could be hampered by the emergence of SCV strains or methicillinresistant strains (especially MRSA), as these are the two most relevant problems. ${ }^{23,24}$ SCV strains can remain undetected if incubation is not prolonged, and this has been recommended for the management of patients with clinical signs and symptoms of infection. ${ }^{25,26}$ Moreover, infection by SCV strains should be considered a possibility if a poor response to treatment is detected, as previously reported in PJIs caused by S. aureus. ${ }^{27}$ The three tested SCV S. epidermidis strains not only required a minimum period of 2 days for growth in conventional media cultures, but also for MIC and MBC determinations, which required a modification of the established microbiological protocols.

In our study, we have detected an elevated number of MRSA (66.67\% of $S$. aureus strains), a fact that confirms the previously reported high prevalence of MRSA. ${ }^{28}$ On the other hand, all the MRSA strains tested exhibited an identical resistance phenotype, which is the commonest one seen in our hospital in recent years (data not shown) and has been previously characterized in Spanish isolates. $^{29}$ This finding suggests that the availability of antimicrobials which are active against MRSA has been drastically reduced. Likewise, a high cloxacillin nonsusceptibility rate was detected among the S. epidermidis strains (68.42\%). 
Table 2 Results of susceptibility studies: $\mathrm{MIC}_{50}, \mathrm{MIC}_{90}\left(\mathrm{mg} \mathrm{I}^{-1}\right)$, nonsusceptibility rate and percentage of bactericidal activity

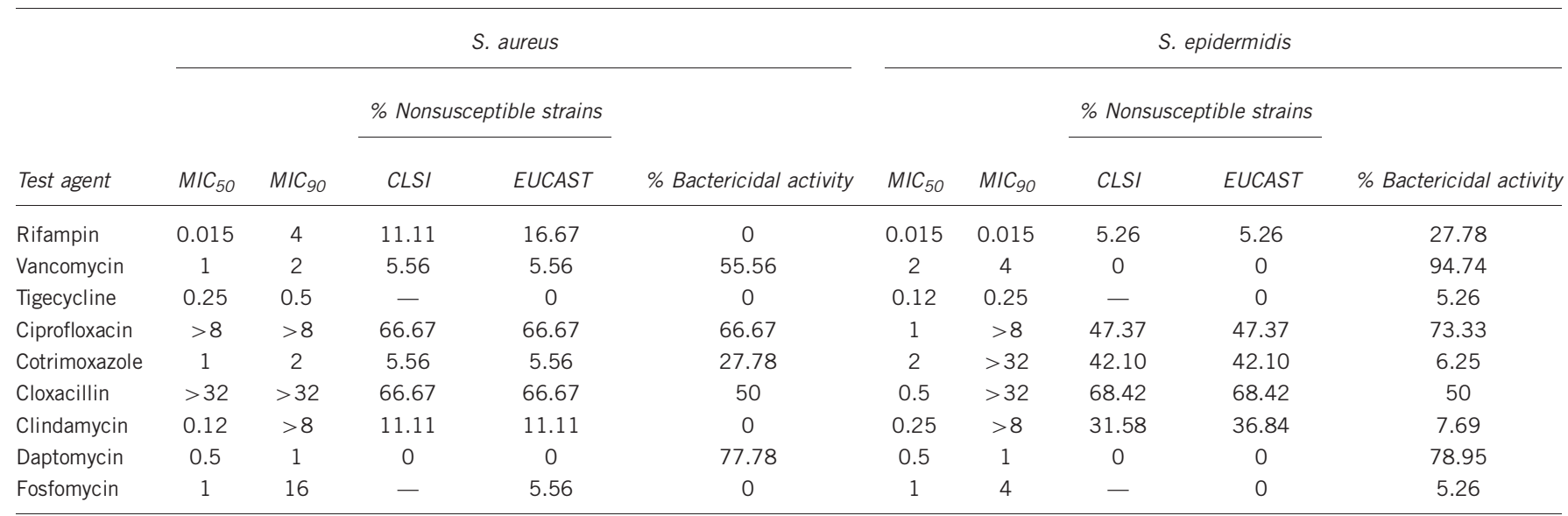

Vancomycin has traditionally been the first-line agent for PJI caused by multidrug-resistant staphylococci strains. However, owing to the recent increase of the MIC values that has been detected in some strains from both species, its use should be undertaken with caution. ${ }^{30}$ In this sense, our study has registered one vancomycin nonsusceptible $S$. aureus strain (5.6\%). On the other hand, the bactericidal activity of vancomycin was higher in S. epidermidis compared with that observed in S. aureus (94.74 vs $55.56 \%$ ), potentially constituting an additional drawback for the use of this antibiotic.

Rifampin, another commonly used antibiotic with outstanding efficacy against these types of clinical isolates, showed high in vitro activity against both staphylococcal species in our series. However, this antibiotic worked as a bacteriostatic agent for most of the tested strains, thus making it necessary to be used in combination with other antimicrobials to avoid resistance development. ${ }^{11,31}$ Tygecicline, daptomycin and fosfomycin could be interesting alternatives for antimicrobial therapy. Our results show that tygecicline inhibited all the tested strains, with no bacterial resistance detected. However, MBC data showed that this antibiotic worked as a bacteriostatic agent against most of the tested strains.

Daptomycin has become a viable i.v. alternative to vancomycin because of its high level of antimicrobial and antibiofilm activity, as previously reported. ${ }^{10,32}$ According to our data, daptomycin not only inhibited all the tested strains but also showed high bactericidal activity for both bacterial species $(77.78 \%$ for S. aureus and $78.95 \%$ for S. epidermidis). These results are in accordance with those of other studies. ${ }^{33}$ However, the need for parenteral administration made daptomycin an alternative only for patients requiring i.v. therapy. Other oral alternatives are therefore necessary for treatment of patients outside the hospital setting. ${ }^{16}$

Finally, fosfomycin seemed to be an efficient agent, with only one $S$. aureus nonsusceptible strain found (5.56\%), although this antibiotic exhibits a mostly bacteriostatic behavior.

Regarding the other tested antibiotics, antimicrobial activity findings generally resulted in differences between both staphylococcal species. Cotrimoxazole, as well as clindamycin, showed a better response against $S$. aureus than against $S$. epidermidis. In fact, all the MRSA strains tested in our study were cotrimoxazole-susceptible. This antibiotic has previously exhibited substantial in vitro activity against most MRSA strains. ${ }^{12}$ Both antimicrobials could be administered orally, thus increasing their attractiveness for the management of some patients.
In conclusion, MRSA strains are an increasingly prevalent cause of PJIs; consequently, the use of more efficient antibiotics is needed. According to our data, rifampin, tigecycline, daptomycin and fosfomycin showed high in vitro activity against most of the tested staphyloccal strains isolated from the PJIs. In vitro results should be considered when determining the therapy for these patients, along with other pathogenic aspects such as biofilm development and prosthesis removal.

\section{ACKNOWLEDGEMENTS}

This work was supported by Comunidad de Madrid (BITI-CAM project S2009/MAT-1472) and Ministerio de Educación y Ciencia (project CONSOLIDER FUNCOAT CSD2008-00023). DMM was funded by the Fundación Conchita Rábago de Jiménez Díaz. AOP and GDP were funded by the Comunidad de Madrid. We acknowledge Mr Oliver Shaw for his help with English language.

1 Lentino, J. R. Prosthetic joint infections: bane of orthopedists, challenge for infectious disease specialists. Clin. Infect. Dis. 36, 1157-1161 (2003).

2 Anagnostakos, K., Schmid, N. V., Kelm, J., Grun, U. \& Jung, J. Classification of hip joint infections. Int. J. Med. Sci. 6, 227-233 (2009).

3 Trampuz, A. \& Zimmerli, W. Prosthetic joint infections: update in diagnosis and treatment. Swiss Med. Wkly. 135, 243-251 (2005).

4 Bernard, L. et al. Trends in the treatment of orthopaedic prosthetic infections. J. Antimicrob. Chemother. 53, 127-129 (2004).

5 Geipel, U. Pathogenic organisms in hip joint infections. Int. J. Med. Sci. 6, 234-240 (2009).

6 Del Pozo, J. L. \& Patel, R. Clinical practice. Infection associated with prosthetic joints. N. Engl. J. Med. 361, 787-794 (2009).

7 Parvizi, J., Azzam, K., Ghanem, E., Austin, M. S. \& Rothman, R. H. Periprosthetic infection due to resistant staphylococci: serious problems on the horizon. Clin. Orthop. Relat. Res. 467, 1732-1739 (2009).

8 Cremniter, J. et al. Decreased susceptibility to teicoplanin and vancomycin in coagulase-negative Staphylococci isolated from orthopedic-device-associated infections. J. Clin. Microbiol. 48, 1428-1431 (2010).

9 Hawser, S. P., Bouchillon, S. K., Hoban, D. J., Dowzicky, M. \& Babinchak, T. Rising incidence of Staphylococcus aureus with reduced susceptibility to vancomycin and susceptibility to antibiotics: a global analysis 2004-2009. Int. J. Antimicrob. Agents 37, 219-224 (2011).

10 Rice, D. A. \& Mendez-Vigo, L. Daptomycin in bone and joint infections: a review of the literature. Arch. Orthop. Trauma Surg. 129, 1495-1504 (2009).

11 Drancourt, M. et al. Oral rifampin plus ofloxacin for treatment of Staphylococcusinfected orthopedic implants. Antimicrob. Agents Chemother. 37, 1214-1218 (1993).

12 Stein, A. et al. Ambulatory treatment of multidrug-resistant Staphylococcus-infected orthopedic implants with high-dose oral co-trimoxazole (trimethoprim-sulfamethoxazole). Antimicrob. Agents Chemother. 42, 3086-3091 (1998).

13 Saginur, R. et al. Multiple combination bactericidal testing of staphylococcal biofilms from implant-associated infections. Antimicrob. Agents Chemother. 50, 55-61 (2006). 
14 Gallo, J. et al. In vitro testing of gentamicin-vancomycin loaded bone cement to prevent prosthetic joint infection. Biomed. Pap. Med. Fac. Univ. Palacky Olomouc. Czech Repub 149, 153-158 (2005).

15 Bassetti, M. et al. Linezolid in the treatment of Gram-positive prosthetic joint infections. J. Antimicrob. Chemother. 55, 387-390 (2005).

16 Esteban, J. \& Cordero-Ampuero, J. Treatment of prosthetic osteoarticular infections. Expert Opin. Pharmacother. 12, 899-912 (2011).

17 Valle, J. et al. SarA and not sigmaB is essential for biofilm development by Staphylococcus aureus. Mol. Microbiol. 48, 1075-1087 (2003).

18 Esteban, J. et al. Evaluation of quantitative analysis of cultures from sonicated retrieved orthopedic implants in diagnosis of orthopedic infection. J. Clin Microbiol. 46, 488-492 (2008).

19 CLSI. Performance Standards for Antimicrobial Susceptibility Testing. Twenty-first informational supplement. In M100-S21 Vol 31 No 1 (2012).

20 EUCAST. Breakpoint tables for interpretation of MICs and zone diameters. In Version 2.0 (2012).

21 Amsterdam, D. Susceptibility testing of antimicrobials in liquid media. In Antibiotics in Laboratory Medicine (Ed., V.L.) 102-103 (Williams \& Wilkins, Philadelphia, 1996).

22 Schito, G. C. The importance of the development of antibiotic resistance in Staphylococcus aureus. Clin. Microbiol. Infect. 12 (Suppl 1) 3-8 (2006).

23 Deurenberg, R. H. et al. The molecular evolution of methicillin-resistant Staphylococcus aureus. Clin. Microbiol. Infect. 13, 222-235 (2007).

24 von Eiff, C. Staphylococcus aureus small colony variants: a challenge to microbiologists and clinicians. Int. J. Antimicrob. Agents 31, 507-510 (2008).
25 Schafer, P. et al. Prolonged bacterial culture to identify late periprosthetic joint infection: a promising strategy. Clin. Infect. Dis. 47, 1403-1409 (2008).

26 Vaudaux, P., Kelley, W. L. \& Lew, D. P. Staphylococcus aureus small colony variants: difficult to diagnose and difficult to treat. Clin. Infect. Dis. 43, 968-970 (2006).

27 Sendi, P. et al. Staphylococcus aureus small colony variants in prosthetic join infection. Clin. Infect. Dis. 43, 961-967 (2006).

28 Kourbatova, E. V. et al. Emergence of community-associated methicillin-resistant Staphylococcus aureus USA 300 clone as a cause of health care-associated infections among patients with prosthetic joint infections. Am. J. Infect. Controll 33, 385-391 (2005).

29 Vindel, A. et al. Methicillin-resistant Staphylococcus aureus in Spain: molecular epidemiology and utility of different typing methods. J. Clin. Microbiol. 47, 1620-1627 (2009).

30 Srinivasan, A., Dick, J. D. \& Perl, T. M. Vancomycin resistance in staphylococci. Clin. Microbiol. Rev. 15, 430-438 (2002).

31 Zimmerli, W., Widmer, A. F., Blatter, M., Frei, R. \& Ochsner, P. E. Role of rifampin for treatment of orthopedic implant-related staphylococcal infections: a randomized controlled trial. Foreign-Body Infection (FBI) Study Group. JAMA 279, 1537-1541 (1998).

32 Picazo, J. J. et al. Comparative activities of daptomycin and several agents against staphylococcal blood isolates. Glycopeptide tolerance. Diagn. Microbiol. Infect. Dis. 70, 373-379 (2011)

33 Sader, H. S., Fritsche, T. R. \& Jones, R. N. Daptomycin bactericidal activity and correlation between disk and broth microdilution method results in testing of Staphylococcus aureus strains with decreased susceptibility to vancomycin. Antimicrob. Agents Chemother. 50, 2330-2336 (2006). 